\title{
120 GBaud PAM-4/PAM-6 generation and Detection by Photonic Aided Digital-to- Analog Converter and Linear Equalization
}

Xin, Haiyun; Kong, Deming; Zhang, Kuo; Jia, Shi; Zhang, Xiaoling; Hu, Weisheng; Hu, Hao

Published in:

Journal of Lightwave Technology

Link to article, DOI:

$10.1109 / j$ lt.2020.2966548

Publication date:

2020

Document Version

Peer reviewed version

Link back to DTU Orbit

Citation (APA):

Xin, H., Kong, D., Zhang, K., Jia, S., Zhang, X., Hu, W., \& Hu, H. (2020). 120 GBaud PAM-4/PAM-6 generation and Detection by Photonic Aided Digital-to-Analog Converter and Linear Equalization. Journal of Lightwave Technology, 38(8), 2226 - 2230. https://doi.org/10.1109/jt.2020.2966548

\section{General rights}

Copyright and moral rights for the publications made accessible in the public portal are retained by the authors and/or other copyright owners and it is a condition of accessing publications that users recognise and abide by the legal requirements associated with these rights.

- Users may download and print one copy of any publication from the public portal for the purpose of private study or research.

- You may not further distribute the material or use it for any profit-making activity or commercial gain

- You may freely distribute the URL identifying the publication in the public portal 


\title{
120 GBaud PAM-4/PAM-6 Generation and Detection by Photonic Aided Digital-to-Analog Converter and Linear Equalization
}

\author{
Haiyun Xin, Deming Kong, Kuo Zhang, Shi Jia, Xiaoling Zhang, Weisheng Hu, and Hao Hu
}

\begin{abstract}
In this paper, for the first time, we propose a photonic-aided digital-to-analog converter (PA-DAC) to break through transmitter-side bandwidth limitation in intensity modulation and direct detection (IM-DD) systems. By using this method, bandwidth of receiver-side devices can be fully utilized, and nonlinear distortions in high-data-rate electrical signals are avoided. Experimental results show that, with only linear equalization, 120 GBaud PAM-4 and PAM-6 signals have been successfully generated and detected with bite error ratios (BERs) below 6.25\%-OH HD-FEC and 20\%-OH SD-FEC limits, respectively.
\end{abstract}

Index Terms-Optical interconnects, Photonic aided digital-to-analog converter, Pulse amplitude modulation, Intensity modulation and direct detection, Linear equalization.

\section{INTRODUCTION}

$\mathrm{W}$ ith the ever-increasing bandwidth demand in datacenter networks (DCN), solutions for high-capacity short-reach interconnects are urgently needed. IEEE has standardized 400 Gigabit Ethernet (GE) in 2018 [1]. It is predicted that the next-generation $800 \mathrm{GE}$ or 1.6 Terabit Ethernet (TE) will arise around 2020 [2]. For intra-datacenter applications, low complexity, low cost, and small footprint are preferred. Thus,

Manuscript received October 15, 2019; revised xx, xx; accepted xx, xx. Date of publication $\mathrm{xx}, \mathrm{xx}$; date of current version $\mathrm{xx}, \mathrm{xx}$. This work was partially supported by the National Natural Science Foundation of China under Grant 61431009, Villum Young Investigator program (2MAC), Villum Fonden (15401) and China Scholarship Council (CSC) under grant 201806230098 (Corresponding author: Haiyun Xin and Hao Hu).

H. Xin is with the Shanghai Institute for Advanced Communication and Data Science, State Key Laboratory of Advanced Optical Communication Systems and Networks, Shanghai Jiao Tong University, Shanghai 200240, China, also with the DTU Fotonik, Technical University of Denmark, Kongens Lyngby 2800, Denmark (email: shepherdgir11238@ sjtu.edu.cn).

D. Kong, S. Jia, and H. Hu are with the DTU Fotonik, Technical University of Denmark, Kongens Lyngby 2800, Denmark (email: dmkon@ fotonik.dtu.dk; shijai@ fotonik.dtu.dk; huhao@ fotonik.dtu.dk )

K. Zhang and W. Hu are with the Shanghai Institute for Advanced Communication and Data Science, State Key Laboratory of Advanced Optical Communication Systems and Networks, Shanghai Jiao Tong University, Shanghai 200240, China (email: cpkuo@ sjtu.edu.cn; wshu@ @jtu.edu.cn)

X. Zhang is with the Key Laboratory of Optical Fiber Sensing and Communications (Education Ministry of China), School of Communication and Information Engineering, University of Electronic Science and Technology of China, Chengdu, Sichuan 611731, China, also with the DTU Fotonik, Technical University of Denmark, Kongens Lyngby 2800, Denmark (email: xizhan@fotonik.dtu.dk). high-capacity intensity modulation-direct detection (IM-DD) systems have attracted a lot of attention. However, the capacity of IM-DD system is mainly limited by the bandwidth of transceivers, and the signal-to-noise ratio (SNR). To handle the bandwidth limitation, powerful digital signal processing (DSP) technologies, such as maximum likelihood sequence estimation (MLSE), faster than Nyquist (FTN), trellis-coded-modulation (TCM) are proposed [3-6]. However, the advanced DSP algorithms exhibit high computational complexity (CC), high power consumption and high cost.

Usually, the bandwidth bottleneck lies in the transmitter side, and the achievable bandwidth of receiver-side devices is two or three times higher than transmitter-side devices. For example, the bandwidth of optical modulators and digital-to-analog converters (DACs) is often half of the bandwidth of photodetectors (PDs) and analog-to-digital converters (ADCs). The bandwidth of receiver-side devices cannot be fully used even if using the powerful DSP algorithms mentioned above. In order to fully utilize the bandwidth of the receiver to increase the overall interconnect capacity, a lot of efforts have been made to achieve a larger transmitter bandwidth. As shown in Table I, methods based on electrical selection [7, 8] and digital band interleaving [9] have been proposed to increase the equivalent bandwidth or sampling rate of the DAC, however, the bandwidth limitation due to electrical drivers and optical modulators still exists.

TABLE I COMPARISON OF HARDWARE REQUIREMENTS AMONG DIFFERENT METHODS FOR TRANSMITTER BANDWIDTH EXTENSION

\begin{tabular}{cccccc}
\hline \hline Ref & Methods & $\begin{array}{c}\text { BW of } \\
\text { electrical } \\
\text { components }\end{array}$ & $\begin{array}{c}\text { DAC } \\
\text { number }\end{array}$ & $\begin{array}{c}\text { BW of } \\
\text { E/O }\end{array}$ & $\begin{array}{c}\text { E/O } \\
\text { number }\end{array}$ \\
\hline$[7]$ & $\begin{array}{c}\text { electrical } \\
\text { selection }\end{array}$ & $\begin{array}{c}\text { high } \\
\text { (Analog Mux) }\end{array}$ & multiple & high & single \\
\hline$[8]$ & $\begin{array}{c}\text { electrical } \\
\text { selection }\end{array}$ & $\begin{array}{c}\text { high } \\
\text { (Analog Mux) }\end{array}$ & multiple & high & single \\
\hline$[9]$ & $\begin{array}{c}\text { digital band } \\
\text { interleaving }\end{array}$ & $\begin{array}{c}\text { high } \\
\text { (Triplexer) }\end{array}$ & multiple & high & single \\
\hline $\begin{array}{c}\text { This } \\
\text { work }\end{array}$ & $\begin{array}{c}\text { Photonic } \\
\text { aided DAC }\end{array}$ & low & multiple & low & multiple \\
\hline \hline
\end{tabular}

In this paper, we propose a photonic-aided digital-to-analog converter (PA-DAC) to break through the overall bandwidth limitation of the transmitter in IM-DD systems. Using an optical pulse source and multiple electro-optical modulators driven by parallel DACs, high baud rate optical signal can be generated without bandwidth limitation from each individual 
device. Equivalently, a higher bandwidth electro-optical DAC is realized. 120 GBaud 4-level and 6-level pulse-amplitude modulation (PAM) signal was generated and successfully detected using the proposed scheme consisting of commercial devices. Only using linear feedforward equalization (FFE), the PAM-4 and PAM-6 signals achieve bit-error ratio (BER) below $6.25 \%$ overhead hard-decision forward error correction (HD-FEC) threshold, and 20\% overhead soft-decision forward error correction (SD-FEC) threshold [10], with the net rates of $224 \mathrm{Gbps}$ and $250 \mathrm{Gbps}$, respectively.

\section{PhOTONIC AIDED DIGITAL-TO-ANALOG CONVERTER}

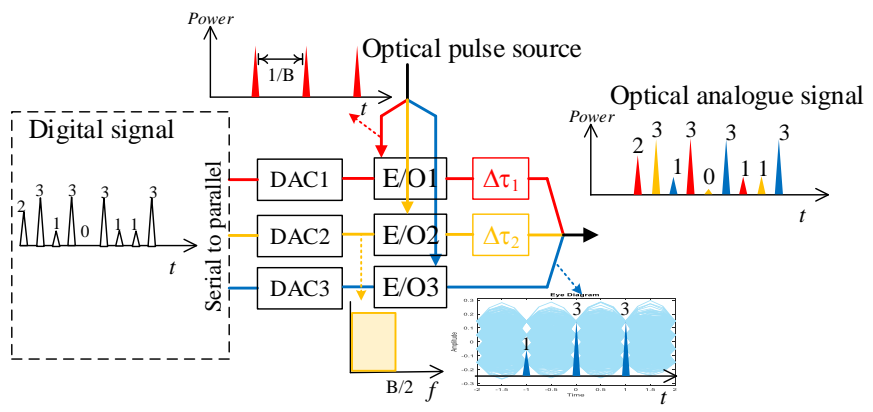

Fig. 1. Principle of photonic-aided digital-to-analog converter.

As illustrated in Fig. 1, the photonic-aided digital-to-analog converter (PA-DAC) consists of an optical pulse source with a repetition rate of $f_{\text {clk }}=B, N$ sub-DACs and $N$ E/O modulators with an analog bandwidth of at least $B / 2$, and passive components including delay lines and splitter/coupler. The target signal sampled at $N^{*} f_{\text {clk }}$ is generated in digital domain, and then loaded into $N$ DACs in parallel. The optical pulse together with $N \mathrm{E} / \mathrm{O}$ modulators works as an optical sampler to the DACs' electrical output. Then, the $N$ branches of optical signals from $\mathrm{E} / \mathrm{O}$ modulators are appropriately delayed and combined, to generate an analog optical signal. In Fig. $1, N$ is set as 3 for convenience of illustration, and it can be arbitrary in practice. Generally, a small $N$ is recommended since less devices introduces less extra cost. In fact, the transmitter configuration of PA-DAC is similar as optical time division multiplexing (OTDM) techniques [12-14]. In those traditional OTDM schemes introduced in direct detection (DD) systems, OTDM de-multiplexing is required in the receiver side, and multiple optical and electrical receivers are needed. In contrast, by using the PA-DAC, the receiver side is same as conventional DD system, where only a single PD and a single ADC are used.

Since InP based electro-absorption modulator (EAM) can be integrated with the monolithically integrated mode-locked laser diode [11], the E/O modulators could potentially be integrated with the pulse source on a single chip. All the passive components, such as splitters, couplers and delay lines, could also be integrated on the same chip. In this case, the optical path lengths of each branch signal will be stable, which only depends on lengths of optical waveguides, and will not be affected by the environmental factors, such as vibration and temperature fluctuation. Thus, random phase variation caused by fibers can be avoided, and devices for high-accuracy timing control are not needed.

Furthermore, since each driving electrical signal has relatively low bandwidth $(\mathrm{B} / 2)$, the nonlinearity caused by drivers and $\mathrm{E} / \mathrm{O}$ modulators is negligible, and computation-consuming nonlinear equalizer can be avoided.

\section{EXPERIMENTAL SETUP AND DSP FLOW}

\section{A. Experimental setup}

Figure 2 (a) illustrates the experimental setup. Considering that the transmitter-side and receiver-side bandwidths are respectively limited to $25 \mathrm{GHz}$ (AWG) and $63 \mathrm{GHz}$ (DSO), the number of sub-DACs and optical modulators is selected as

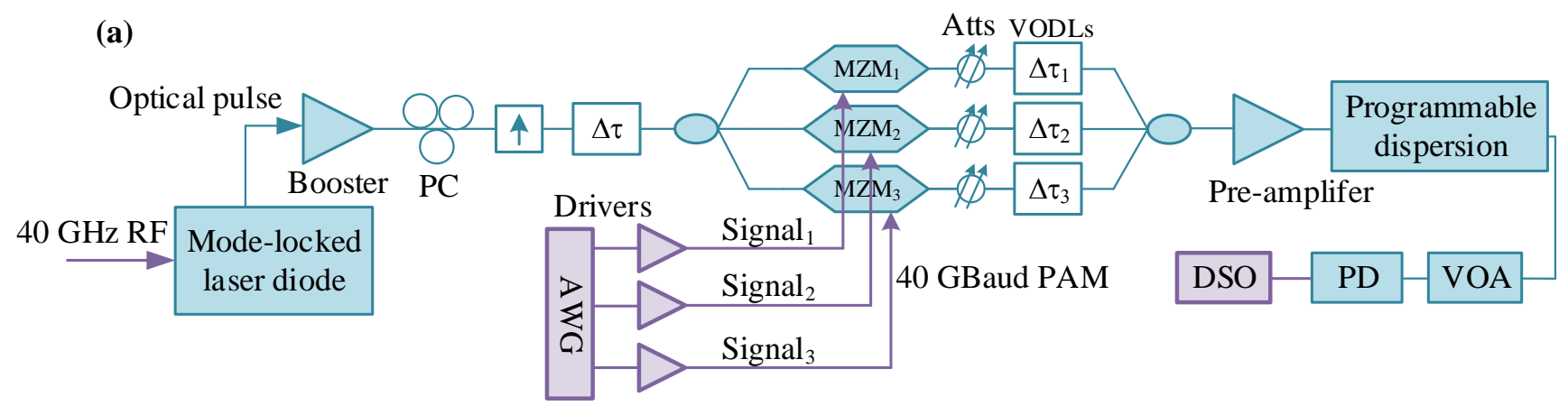

(b)

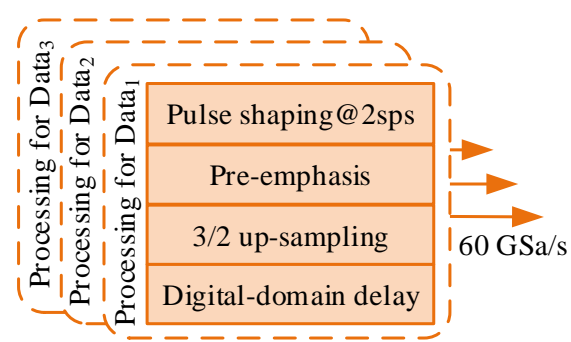

(c)

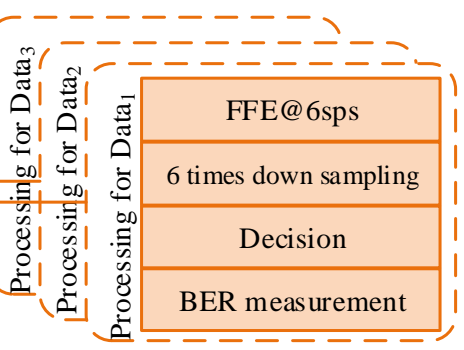

Fig. 2(a): Experimental setup. (b): DSP flow in transmitter side, (c): DSP flow in receiver side. 
three. The short optical pulse with a pulse width of $\sim 2 \mathrm{ps}$ is generated by a monolithically integrated mode-locked laser diode (MLLD), which is driven by a $40 \mathrm{GHz}$ radio frequency (RF) signal with a power of $15 \mathrm{dBm}$. Correspondingly, the period of optical pulse is 25 ps. After the amplification, polarization control and delay adjustment, the optical pulse signal is split into three portions and then launched into three Mach-Zehnder modulators (MZM). Each modulator is driven by a 40-GBaud PAM signal. For optical delay line adjusting and power equalization, we used a PAM-2 signal for convenience. By properly adjusting $\Delta \tau$ and electrical delays of the three signals, the optical pulse is aligned to the decision point of PAM-2 signals in each branch. Then, the three PAM-2 modulated optical signals are adjusted in time by variable optical delay lines $\left(\Delta \tau_{1}, \Delta \tau_{2}, \Delta \tau_{3}\right)$ to make the optical pulses distribute uniformly in time domain, and by variable optical attenuators (VOAs) to equalize the power of the three optical signals. Then, we respectively applied PAM-4 and 2D PAM-6 for BER performance testing. Since the neighbouring branches do not overlap in time, the three 40 GBaud PAM-4/PAM-6 signals are combined into one 120 GBaud PAM-4/PAM-6 signal by optical couplers. After the amplification, a programmable dispersive element is used to emulate the fiber dispersion and variable optical attenuator (VOA) is used to adjust the input power of the photodiode (PD, XPDV3120R) with a $70-\mathrm{GHz}$ bandwidth. Finally, the electrical signal is captured by a digital signal oscilloscope (DSO, Keysight, DSA-X 93304Q) with a bandwidth of $63 \mathrm{GHz}$ and a sampling rate of $160 \mathrm{GSa} / \mathrm{s}$.

\section{B. Transmitter-side DSP flow}

Figure 2 (b) shows the DSP flow in the transmitter side. After PAM mapping, the signal is pulse shaped by a raise cosine filter with a roll-off factor of 0.01 , such that the required bandwidth of DAC and MZM is only $20.2 \mathrm{GHz}$. Then, we apply digital pre-emphasis using the method reported in [15], to compensate the response penalty of transmitter-side devices, including AWG, driver, electrical attenuator and optical modulator. Appropriate delays are also applied by digital interpolation to guarantee that the decision point of each PAM signal is aligned to the optical pulse. Afterwards, the signal is re-sampled to match the sampling rate of AWG $(60 \mathrm{GSa} / \mathrm{s})$. Finally, three-branch PAM signals at 40 GBaud are obtained.

\section{Receiver-side DSP flow}

Figure 2 (c) shows the DSP flow in the receiver side. Firstly, the received signal is re-sampled. Considering that interval errors between optical pulses are difficult to avoid, we adopt three parallel equalizations respectively for three 40-GBaud PAM signals rather than one equalization for one 120-GBaud PAM signal. The inter symbol interference (ISI) can be divided into inter branch ISI and intra branch ISI. The inter branch ISI refers to the interference among adjacent 120 GBaud PAM symbols. It mainly occurs after PD detection and digitization of DSO, and is caused by non-ideal response of PD and ADC. This inter branch ISI can be mitigated by a simple linear equalizer, which is a feed forward equalizer (FFE) of $240 \mathrm{GSa} / \mathrm{s}$ (2sps for 120 GBaud signal, 6sps for 40 GBaud signal), and applied to the received signal. In order to distinguish the three branches of signal, we respectively set different original symbols as the training sequence. The coefficients are updated every 6 samples using least mean square (LMS) algorithm. In addition to the ISI incurred by receiver filtering effect, slight interference might occur between optical pulses, causing fluctuation of direct current (DC) offset and gain of the received electrical signal. Thanks to the adaption of DC component and gain coefficient in FFE, the fluctuation can be well tracked. On the other hand, intra branch ISI refers to the interference among adjacent 40 GBaud PAM symbols within each branch. It mainly occurs in the transmitter side before optical coupling, which is caused by non-ideal response of transmitter-side devices, including DAC, driver and MZM. The linear part can be eliminated by the pre-emphasis filter of transmitter side. But the nonlinear part caused by sine mapping of MZM is left. For the purpose of reducing computational complexity (CC), the corresponding equalizer is working at $1 \mathrm{sps}$, and applied to each branch of PAM signal. Finally, demodulation is performed for bit error ratio (BER) measurement.

\section{RESUlTS AND DisCUSSIONS}

\section{A. Optical pulses before and after modulation}
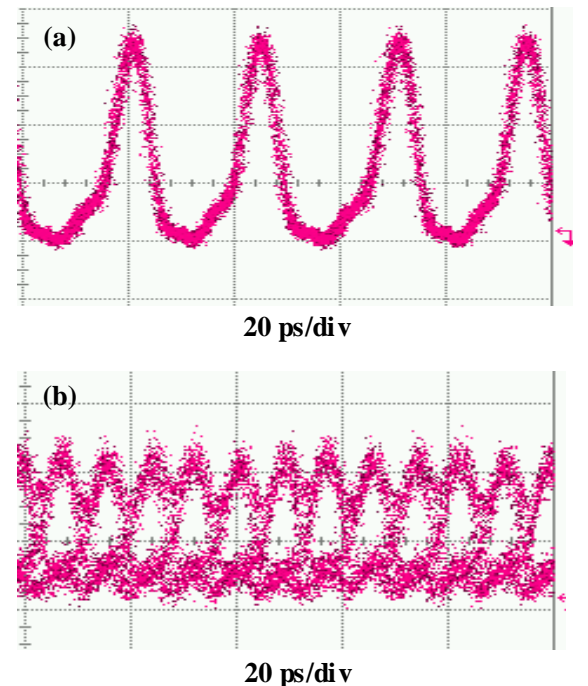

Fig. 3 (a) Optical pulses of $40 \mathrm{GHz}$; (b) The generated 120 GBaud OOK signal. Resolution: 20ps/div.

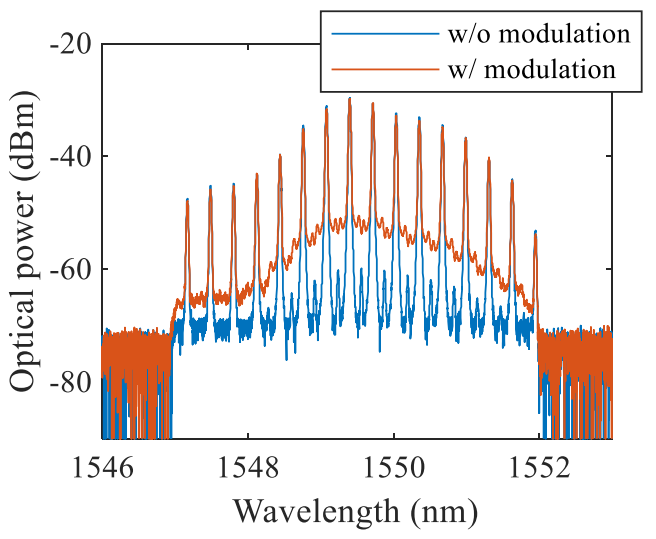

Fig. 4. Spectra of optical pulses before and after modulation. 
Figure 3(a) shows the eye diagram of the $40-\mathrm{GHz}$ optical pulses. Due to the limited bandwidth $(70 \mathrm{GHz})$ of the sampling oscilloscope, the optical pulse in Fig. 3(a) looks broader than the actual pulse with the pulse width of $\sim 2$ ps. For the convenience of delay and power adjustment, we generate a 120 GBaud PAM-2 signal using this optical pulse, with the eye diagram shown in Fig. 3(b). The spectra of optical pulses before and after the modulation are respectively shown in Fig. 4.

B. Performance evaluation for 120 GBaud PAM-4

(a)

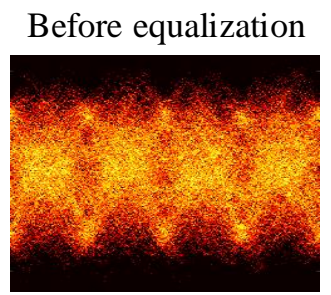

After equalization

(b)

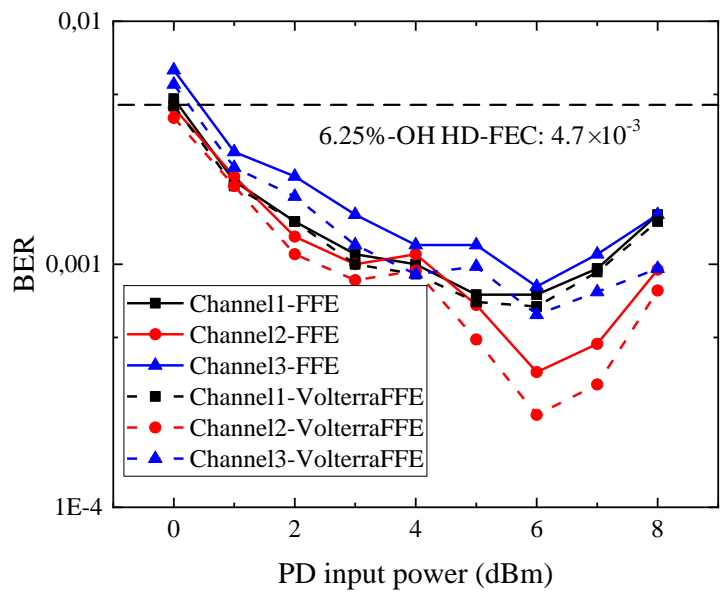

(c)

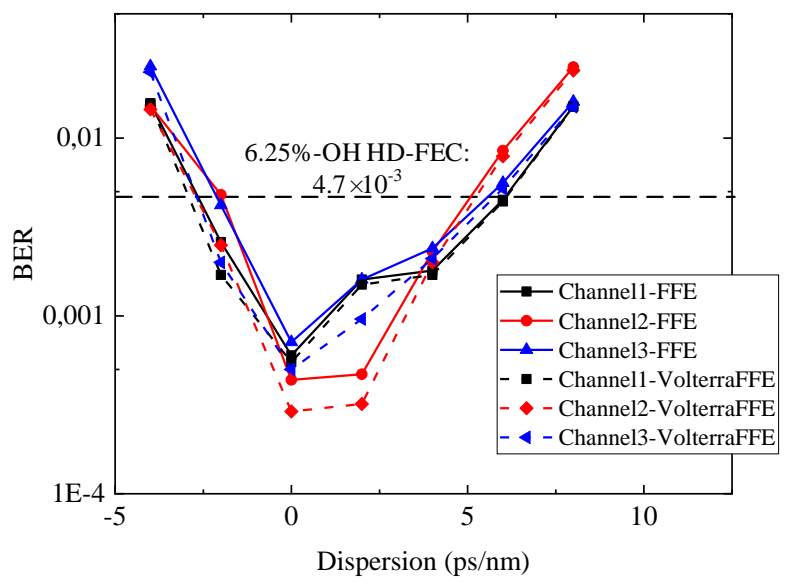

Fig. 5. Results of PAM-4: (a) Eye diagrams before and after equalization; (b) BER performance as a function of PD input power; (c) BER performance as a function of dispersion.

The results of each branch PAM-4 signal after the FFE and Volterra-FFE are shown in Fig. 5. Eye diagrams of PAM-4 signal before and after equalization are shown in Fig. 5(a). Figure 5(b) shows the BER performance as a function of PD input power. Hard decision-forward error correction (HD-FEC) limit can be achieved by using only FFE, and the performance improvement by using Volterra FFE is not much. Figure 5(c) shows the BER performance as a function of dispersion. As can be seen, performance degradation is caused by negative or positive dispersion. It is because that, optical pulse is broadened by the dispersion, and the interference between neighbouring pulses become more serious. The tolerant dispersion value is from -2ps to $5 \mathrm{ps}$, considering the limit of 6.25\%-OH HD-FEC.

\section{Performance evaluation for 120 GBaud PAM-6}

(a)

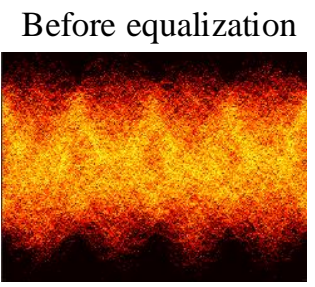

After equalization

(b)

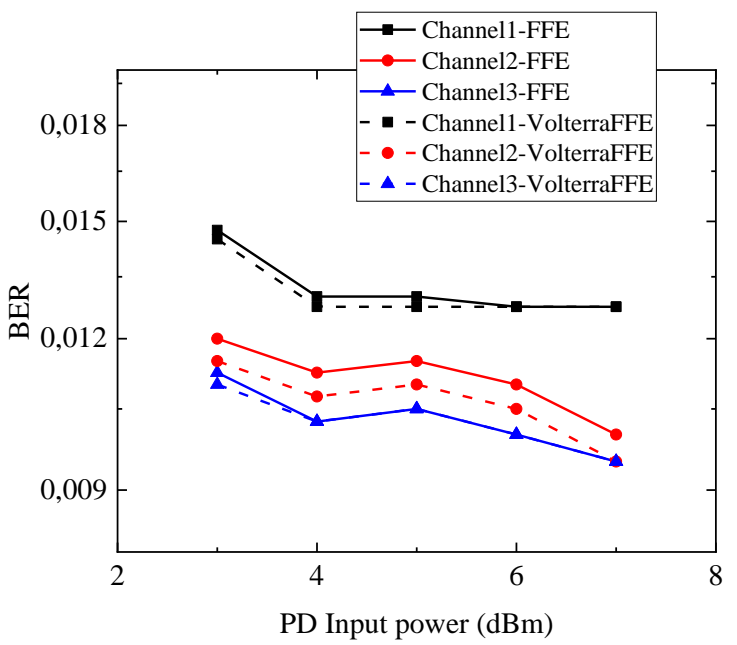

(c)

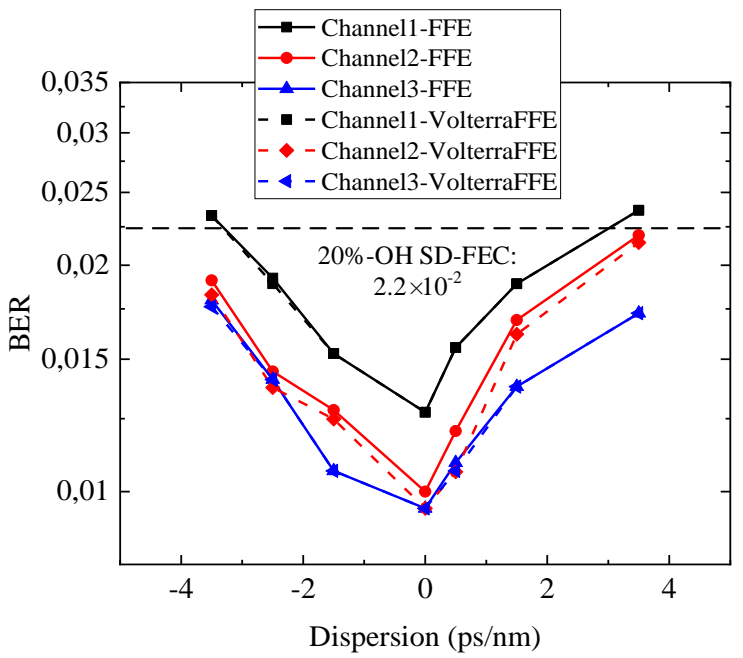

Fig. 6. Results of PAM-6: (a) Eye diagrams of PAM-6 signal before and after equalization (b) BER performance as a function of PD input power; (c) BER performance as a function of dispersion.

The results of each branch PAM-6 signal after the FFE and Volterra-FFE are shown in Fig. 6. Here, we used the suboptimum encoding algorithm in [16]. Two consecutive PAM-6 symbols constitute 36 constellation points in 2D space, and four outer ones are deleted such that 32 constellation points 
are left to represent 5 information bits. Thus, each symbol carries 2.5 bits. In Fig. 6(a), eye diagrams of 2D-PAM-6 before and after equalization are illustrated. Figure 6(b) shows the BER performance as a function of PD input power. $20 \%-\mathrm{OH}$ SD-FEC $\left(2.2 \times 10^{-2}\right)$ limit can be achieved by using only FFE and again the performance improvement by using the Volterra FFE is not much. As shown in Fig. 6(c), the tolerant dispersion value is from -3 ps to $3 \mathrm{ps}$, considering the limit of $20 \%-\mathrm{OH}$ SD-FEC.

\section{CONCLUSIONS}

In this paper, we have proposed and demonstrated photonic aided digital-to-analog converter in the conventional IM-DD system to break through transmitter-side bandwidth limitation. Since each driving electrical signal has relatively low frequency, serious nonlinear distortions caused by electrical components can be avoided. Experimental results show that, by only using linear equalization, 120 GBaud PAM-4 and PAM-6 signals have been successfully generated and detected with the BERs below $6.25 \%$-OH HD-FEC and 20\%-OH SD-FEC limits, respectively.

\section{ACKNOWLEDGMENT}

The authors would like to thank Fraunhofer HHI, Berlin, Germany, for kindly providing the monolithic integrated mode-locked laser diode.

\section{REFERENCES}

[1] IEEE P802.3bs $400 \mathrm{~Gb} / \mathrm{s}$ Ethernet Task Force, 2018. http://www.ieee802.org/3/bs/

[2] J. L. Wei, Q. Cheng, R. V. Penty, I. H. White, and D. G. Cunningham, "400 Gigabit Ethernet using advanced modulation formats: Performance, complexity, and power dissipation,"IEEE Commun.Mag.,vol. 53, no. 2, pp. 182-189, Feb. 2015.

[3] Q. Hu, K. Schuh, M. Chagnon, F. Buchali, and H. Bülow, "Up to 94 GBd THP PAM-4 transmission with $33 \mathrm{GHz}$ bandwidth limitation," in Proceedings of European conference on optical communications (ECOC, 2018), paper Th3F.6.

[4] A. Masuda, S. Yamamoto, H. Taniguchi, M. Nakamura, and Y. Kisaka, "255-Gbps PAM-8 transmission under 20-GHz bandwidth limitation using NL-MLSE based on Volterra filter," in Optical Fiber Communication Conference (OFC, 2019), paper W4I.6.

[5] N. Stojanovic, C. Prodaniuc, L. Zhang, J. Wei, S. Calabrò, and T. Rahman, and C. Xie, "4D PAM-7 trellis coded modulation for data center," IEEE Photon. Technol. Lett., vol. 31, no. 5, pp. 369-372 (2019)

[6] S. Yamamoto, A. Masuda, H. Taniguchi, and Y. Kisaka, "92-Gbaud PAM4 transmission using spectral-shaping trellis-coded-modulation with 20-GHz bandwidth limitation," in Optical Fiber Communication Conference (OFC, 2019), paper W4I.5.

[7] H. Yamazaki et al., "Discrete multi-tone transmission at net data rate of $250 \mathrm{Gbps}$ using digital-preprocessed analog-multiplexed DAC with halved clock frequency and suppressed image,"J. Lightw.Technol., vol. 35,no. 7, pp. 1300-1306, Apr. 2017.

[8] H. Mardoyan, F. Jorge, O. Ozolins, J. M. Estaran, A. Udalcovs, A. Konczykowska, M. Riet, B. Duval, V. Nodjiadjim, J. Dupuy, X. Pang, U. Westergren, J. Chen, S. Popov, and S. Bigo, "204-GBaud On-Off Keying Transmitter for Inter-Data Center Communications," in Optical Fiber Communication Conference (OFC, 2018), paper Th4A.4.

[9] X. Chen, S. Chandrasekhar, J. Cho, and P. Winzer, "Single-Wavelength and Single-Photodiode Entropy-Loaded 554-Gb/s Transmission over 22-km SMF," in Optical Fiber Communication Conference (OFC, 2019), paper Th4B.5.

[10] L. M. Zhang and F. R. Kschischang, "Staircase codes with $6 \%$ to $33 \%$ overhead," J. Light. Technol. vol. 32, no. 10, pp. 1999-2002, 2014.
[11] R. Kaiser and B. Huttl, "Monolithic 40-GHz mode-locked MQWDBR lasers for high-speed optical communication systems," IEEE J. Sel. Topics Quantum Electron., vol. 13, no. 1, pp. 125-135, Jan.-Feb.2007.

[12] M. Nakazawa, T. Yamamoto, and K.R. Tamura, "1.28Tbit/s-70km OTDM transmission using third- and fourth-order simultaneous dispersion compensation with a phase modulator," Electronics Letters, vol. 36, no. 24 (2000)

[13] T. Hirooka, R. Hirata, J. Wang, M. Yoshida, and M. Nakazawa, "Single-channel $10.2 \mathrm{Tbit} / \mathrm{s}$ (2.56 Tbaud) optical Nyquist pulse transmission over 300 km," Opt. Exp., vol. 26, no. 21 27221-27236, Oct. 2018.

[14] H. Hu, H. C. H. Mulvad, C. Peucheret, M. Galili, A. Clausen, P. Jeppesen, and L. K. Oxenløwe, "10 GHz pulse source for $640 \mathrm{Gbit} / \mathrm{s}$ OTDM based on phase modulator and self-phase modulation," Opt. Exp., vol. 19, no. 26, pp. B343-B349, Oct. 2011.

[15] K. Zhang, Q. Zhuge, H. Xin, Z. Xing, M. Xiang, S. Fan, L. Yi, W. Hu, and David V. Plant, "Design and analysis of high-speed optical access networks in the O-band with DSP-free ONUs and low-bandwidth optics," Opt. Exp., vol. 21, no. 21, pp. 27873-27884, Dec. 2018.

[16] N. Stojanovic et al., "210/225 Gbit/s PAM-6 transmission with BER below KP4-FEC/EFEC and at least $14 \mathrm{~dB}$ link budget," in Proceedings of European conference on optical communications (ECOC, 2018), paper We1H.5. 